\title{
Mathematical Model for Laboratory System of Bioluminescent Whole-Cell Biosensor with Optical Element
}

\section{Kalabova $\mathrm{H}^{1 *}$, Pospisilova $\mathbf{M}^{1}$, Jirina $\mathbf{M}^{2}$ and Kuncova $\mathbf{G}^{3}$}

${ }^{1}$ Faculty of Biomedical Engineering, Czech Technical University in Prague, Czech Republic

${ }^{2}$ Faculty of Information Technology, Czech Technical University in Prague, Czech Republic

${ }^{3}$ Institute of Chemical Process Fundamentals of the Czech Academy of Sciences, Czech Republic

\begin{abstract}
The fiber-optic biosensor with encapsuled bioreporters use a special optical element (OE) from pure silica with an active layer contents bioluminescent bioreporters was developed as a real in-situ detector for on-line measurement in remote localities. The active layer of biosensor contents bioluminescent bioreporters - genetic modified cells, which are sensitive to its surrounding environment-immobilized in silica matrix on the end of OE. Bioreporters are able to react by emission of visible light $(\approx 500 \mathrm{~nm})$ - bioluminiscence reaction $(B L)$ - in presence of specific analyte.

The genetic modified bacterial stain Pseudomonas putida TVA8 was chosen as biorecognition part of biosensor producing $B L$ in the presence of benzene, toluene, ethylbenzene and xylens (BTEX). Very low level of BL signal was detected by high-sensitive photon-counter. The sensitivity of biosensor depends on a value of detected BL and it can be very low. The signal is also affected by number of cells (light sources) immobilized into the active layer and transmission through OE. Mathematical model of OE shape was developed based on geometric optics. The numerical model is built around the MATLAB scripting language. Simulation of ray transmission was calculated for OE with different shape.
\end{abstract}

Keywords: Biosensor; Optical element; Bioreporter; Bioluminescence

\section{Introduction}

Optical fiber biosensors as instruments for simple, rapid and continuous in-situ monitoring techniques have been intensively investigated [1,2]. Bioactive sensing layer (BSL) (as one of the base parts of biosensor) with transducer provides output optical signal under certain conditions. This optical signal brings information about changes in BSL caused by changes of chemical or physical properties in its close surrounding. BSL is realized by immobilizing cells - bioluminescent bioreporters $(\mathrm{BB})$ - which are able produce bioluminescence $(\mathrm{BL})$ in contact with a specific substance. BL is light emitted in visible spectral range by biological systems arising from enzyme-catalyzed chemical reactions [3-5]. For our purpose, BB represents point light sources which are immobilized in matrices from suitable material [6]. The performance of the biosensorsystem was examined as a function of following parameters: a) gel probe matrix volume, b) bacterial cell density and c) numerical aperture (NA) of the fiber core.

$\mathrm{BL}$ produced by $\mathrm{BB}$ in contact with pollutants is too low what limits sensitivity of biosensor especially in case of tiny optical fiber sensors. Two basic factors contributing to effectively to collect of BL into optical fiber were defined: 1) light transport from its source via optic element (OE) to the detector and 2) light coupling to the $\mathrm{OE}$, which depends on angular distribution of photons entering the fiber what has been investigated by using Monte-Carlo - corrected diffusion method [7]. The authors founded that the coupling efficiency of light was highly depended on the numerical aperture (NA) of the optical fiber. For miniaturization of optical fiber sensors for measurements of $\mathrm{pH}$, oxygen in living cells, DNA characterization and increasing of evanescence-wave effect tapered fibers have been tested in literature $[8,9]$.

In previous study we calculated coupling light efficiency from point light sources into PCS fiber in value $\mathrm{x}=3.5 \%$, which means that only $3.5 \%$ of light emitted by cells is coupled and guided from the cell layer to the detector [10].
The effort our investigation was therefore focused on increasing of the detected BL by increasing of number of $\mathrm{BB}$ and an optimization of OE shape. Number of BB depends on an area where BB are applied. The taper optical element $(\mathrm{OE})$ increase this area compared with PCS fiber, what enables to increase of number of $\mathrm{BB}$.

The maximum diameter of PCS fiber core, to maintain fiber flexibility, is $600 \mu \mathrm{m}$. Using of tapered OE spliced with such optical fiber is the way, how to increase the area covered with cells. With regards that attenuation of splice of two PCS fibers by commercial splicing machines is about $0.02 \mathrm{~dB}$ [11] employment of $\mathrm{OE}$ in design of optical fiber biosensors might overcome troubles with detection of low intensities of bioluminescence produced by bioluminescent bioreporters.

In this paper BL coupling into PCS fiber and into tapered OEs with the various shapes was compared with developed mathematical model for optimizing of tapered $\mathrm{OE}$ shape. The coupling efficiency and results from mathematical model was demonstrated with the selective BB, Pseudomonas putida TVA8 immobilized in active layer by entrapment into silica gel on the tip of PCS fiber and OE.

The proposed biosensor design is a tool for simple, cheap, fast and continuous environmental pollution monitoring. This arrangement can also be used for the construction of biosensors for the detection of

*Corresponding author: Kalabova $\mathrm{H}$, Faculty of Biomedical Engineering, Czech Technical University in Prague, Czech Republic, Tel:+420 224351 111; E-mail hana.kalabova@fbmi.cvut.cz

Received January 30, 2018; Accepted February 16, 2018; Published February 23, 2018

Citation: Kalabova H, Pospisilova M, Jirina M, Kuncova G (2018) Mathematical Model for Laboratory System of Bioluminescent Whole-Cell Biosensor with Optical Element. J Biosens Bioelectron 9: 250. doi: 10.4172/2155-6210.1000250

Copyright: @ 2018 Kalabova H, et al. This is an open-access article distributed under the terms of the Creative Commons Attribution License, which permits unrestricted use, distribution, and reproduction in any medium, provided the original author and source are credited. 
different analytes, depending on the type of bioreports chosen, with a prerequisite for their use in diagnostic and medical applications.

\section{Materials and Methods}

All chemicals were commercial grade supplied by Aldrich, Fluka, Aplichem and Oxoid. Polymer cladded optical fibers (PCS), produced in Laboratory of Optical Fibers of the Institute of Photonic and Electronics, Czech Academy of Sciences, had quartz core $\left(\varnothing_{\text {core }}=600 \mu \mathrm{m}\right.$, $\left.\mathrm{n}_{\text {core }}=1.457\right)$, and silicone polymer cladding $\left(\varnothing_{\text {clad }}=800 \mu \mathrm{m}, \mathrm{n}_{\text {clad }}=1.41\right)$.

\section{Optical element}

The tapered OE was obtained as part of quartz preform scrap from drawing of PCS fibers with the ends polished to the optical quality for the cell immobilization. The OEs were characterized by diameters of both ends $\left(d_{\text {min }}, d_{\max }\right)$, and length $l=z_{\max }$ (Figure 1). Diameters $\left(d_{i}\right)$ of each OE were measured in the $5 \mathrm{~mm}$ distances in $\mathrm{z}$ axis between the ends for calculation (approximation) of analytical function of its shape (Table 1).

\section{Cells}

Pseudomonas putidaTVA8 is capable of producing BL selectively in the presence of benzene, toluene, ethylbenzene and xylenes (BTEX) [12]. The genetically modified bacteria utilized a genetic construct consisting of the lux gene cassette derived from the marine bacterium Vibrio fischeri[13]. The bioreporter was kindly delivered from the University of Tennessee, Knoxville, USA.

Cultivation of Pseudomonas putida TVA8 and its entrapment into silica gel were detail described by authors [14].

\section{BL measurement}

PCS fibers and OEs were connected by SMA optical fiber bare connector to a detector (photon-counter, Perkin-Elmer 3954-P-087). The BL intensity of the induced cells in the active layers was recorded each $10 \mathrm{~s}$. All measurements were realized inside a dark box.

\section{Results and Discussion}

\section{Mathematical model}

For the development of the mathematical model of OE was hypothesized an assumption that the cells are organized in one layer,

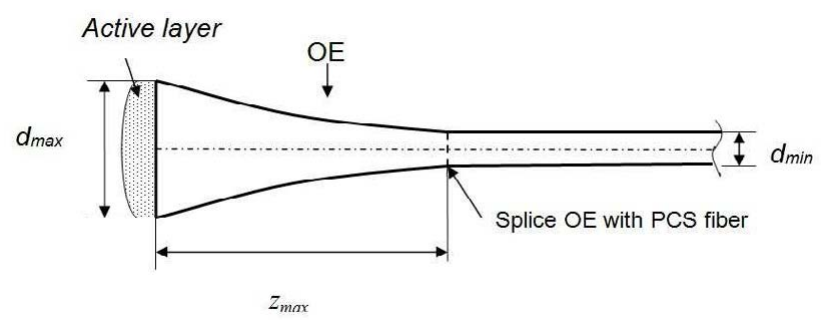

Figure 1: The scheme of tapered OE with an active layer. cell has a sphere-like shape with diameter $1 \mu \mathrm{m}$ and cell is point source, which intensity decreases with quadrate of distance. Methods of geometric optic were applied for calculation of the efficiency of BL coupling into OEs. The OE is cylindrical symmetry body with rotation in $z$ axis (Figure 1).

When optimizing the shape of OE was build on the mathematical model and was looked for such (optimal) set of parameters to maximize the flow of light through the OE from its broad origin that is covered by bioluminescent cells to its narrower tail end with aphotosensitive sensor. The geometric model of $\mathrm{OE}$ is similar to a truncated cone with the difference that the surface curve is not a straight line, but is given by equation of OF shape (eqn. (1)).

$$
\frac{d(z)}{2}=r_{o}+A_{1} e^{\frac{-z}{t_{1}}}+A_{2} e^{\frac{-z}{t_{2}}}
$$

Parameters to be optimized are $r_{0}, A_{1}, t_{1}, A_{2}$ and $t_{2}$ and the total length $\mathrm{z}_{\max }$ of the OE.

To find the optimal values of these parameters, was used the method of ray tracing. The beams are repeatedly cast in appropriate directions given by the angle of aperture and if they approach the tail end of the OE the angle of apeeture is evaluated again to decide wheter the bean reflect from the tail end or passes through. The size of the angle of aperture depends on the refractive index of the material of the $\mathrm{OE}$ and the surrounding environment. Rays that are oriented within the angle of aperture pass through the $\mathrm{OE}$, the others are reflected back. A similar situation occurs when beams gets off of the tail end of the OE. It is fulfilled if the beam falls into the range of the angle of aperture as well.

The objective function of our optimization problem, which is to minimize, is a ration of the number of failed beams, i.e., those that have undergone another way into the environment than through the tail end of the $\mathrm{OE}$, to the total number of rays sent.

First, randomly (stochastically) or systematically (deterministically) was generated a place at the origin round part and second randomly or systematically was generated a directional vector in the corresponding angle of aperture. The directional vector is determined by two randomly generated angles (azimuth and elevation). The azimuth ranges from 0 to $2 p$ and the elevation (measured from upright) rages from 0 to $N A / 2$. By the conversion from spherical to Cartesian coordinates, was obtained the direction vector of the beam.

To simplicity the task was assumed that all bioluminescence cells behave the same, i.e., they emit light of equal intensity within equally probable angle, size of each cell is $1 \mathrm{~mm}$ and the cells are organized in a triangular mesh.

The coasted beam is reflected off the walls or from the origin or tail end of the OE. The reflection is solved using vector calculus in space. First, the intersection of the beam with the wall of the OE is solved. The best solution is to express a directional beam and the OE surface by parametric equations. Parametric vector equation of the line is

\begin{tabular}{|c|c|c|c|c|c|c|c|c|}
\hline & $\begin{array}{l}{ }^{d} \max \\
{[\mathrm{mm}]}\end{array}$ & $\begin{array}{l}{ }^{d} \min \\
{[\mathrm{mm}]}\end{array}$ & $\begin{array}{l}{ }^{z} \max \\
{[\mathrm{mm}]}\end{array}$ & $\begin{array}{c}r^{\prime} \\
{[\mathrm{mm}]}\end{array}$ & $\begin{array}{c}{ }^{A 1} \\
{[\mathrm{~mm}]}\end{array}$ & $\begin{array}{c}{ }^{t} \\
{[\mathrm{~mm}]}\end{array}$ & $\begin{array}{c}{ }^{A} 2 \\
{[\mathrm{~mm}]}\end{array}$ & $\begin{array}{c}{ }^{t} \\
{[\mathrm{~mm}]}\end{array}$ \\
\hline OE 1 & 6.29 & 0.83 & 320 & $0.27 \pm 0.03$ & $1.73 \pm 0.08$ & $33.78 \pm 1.37$ & $1.12 \pm 0.06$ & $154.60 \pm 18.26$ \\
\hline OE 2 & 5.97 & 0.32 & 63 & $0.11 \pm 0.01$ & $2.87 \pm 0.01$ & $14.53 \pm 0.17$ & 0 & 0 \\
\hline OE 3 & 5.06 & 0.89 & 190 & $0.42 \pm 0.01$ & $2.12 \pm 0.01$ & $50.77 \pm 0.70$ & 0 & 0 \\
\hline OE 4 & 9.66 & 0.78 & 170 & $0.26 \pm 0.03$ & $2.78 \pm 0.19$ & $17.23 \pm 0.88$ & $1.81 \pm 0.17$ & $66.46 \pm 8.00$ \\
\hline
\end{tabular}

Table 1: Parameters of optical elements (OE) 
$\vec{p}=A+t . \vec{s}$

or expressed by individual components

$p_{x}=A_{x}+t \times s_{x}$

$p_{y}=A_{y}+t \times s_{y^{\prime}}$

$p_{z}=A_{z}+t \times s_{z}$.

where $\vec{p}=\left(p_{x}, p_{y}, p_{z}\right)$ represents a point on the line, $\mathrm{A}=\left[\mathrm{A}_{\mathrm{x}}, \mathrm{A}_{\mathrm{y}}, \mathrm{A}_{z}\right]$ is the point on the line that corresponds to the initial point of entry on the origin of the OE the beam is spread from, $\vec{s}=\left(s_{x}, s_{y}, s_{z}\right)$ is the directional vector of the beam and $\mathrm{t} \in \mathrm{R}$ is the parameter of the line.

The parametric expression of the model of the OE surface is

$o_{x}=r(z) \cdot \cos \phi$,

$o_{y}=r(z) \cdot \sin \phi$,

$o_{z}=z$,

where $\phi$ is the angle between 0 and $2 \pi, z$ is a parameter expressing a position on the axis of $\mathrm{OE}$ and $r(z)$ the radius according to the equation above, which expresses the current radius of $\mathrm{OE}$ in a given location along the axis of the $\mathrm{OE}$.

The intersection of the beam with the OE surface we get by comparing the parametric equations of the line and the model of the OE surface. After substitution for $r(z)$ was obtained the relations

$$
\begin{aligned}
& A_{x}+\text { t. } s_{x}=\left(r_{0}+A_{1} \cdot e^{-z / t_{1}}+A_{2} \cdot e^{-z t_{2}}\right) \cdot \operatorname{Cos} \varphi \\
& A_{x}+\text { t. } s_{x}=\left(r_{0}+A_{1} \cdot e^{-z / t_{1}}+A_{2} \cdot e^{-z t_{2}}\right) \cdot \operatorname{Sin} \varphi \\
& A_{z}+\text { t. } s_{z}=z
\end{aligned}
$$

for unknown parameters $t, \phi$ and $z$, from which we can calculate the intersection $P$ by re-substituting parameter $t$ into the above equations for the line or re-substituting parameters $\phi$ and $z$ into the above equations for the OE. Solution in a closed analytical form does not exist so the system of equations needs to be solved numerically. When proper choice of initial conditions (the estimated point intersection) is selected the solution can be found quickly and without doubt about correct solution.

The next step is to find the normal vector of $\mathrm{OE}$ at the intersection P. Parametric equations of the normal $\vec{n}=\left(n_{x}, n_{y}, n_{z}\right)$ are

$$
\mathrm{n}_{\mathrm{x}}=\mathrm{P}_{\mathrm{x}}+\mathrm{k} \cdot\left(\mathrm{P}_{\mathrm{x}}-0\right) \mathrm{n}_{\mathrm{y}}=\mathrm{P}_{\mathrm{y}}+\mathrm{k} \cdot\left(\mathrm{P}_{\mathrm{y}}-0\right), \mathrm{n}_{\mathrm{z}}=\mathrm{P}_{\mathrm{z}}+\mathrm{k} \cdot\left[\mathrm{P}_{\mathrm{z}}-\mathrm{r}(\mathrm{z}) \cdot \dot{\mathrm{r}}(\mathrm{z})\right]
$$

where $k$ is a parameter of the line, $k \in R$.

The last step is to find the direction vector of the reflected ray. Mathematical reflection can be utilized. The directional vector of the reflected beam is

$$
\vec{q}=P+\vec{v}-2 \cdot(\vec{v} \cdot \vec{u}) \cdot \vec{u},
$$

Where $\vec{u}=\vec{n} /\|\vec{n}\|$ is the unit normal vector, $\vec{v}=P-A$ and $(\vec{v} \cdot \vec{u})$ is the dot product of the vectors.

The result is an equation of the line representing the reflected beam, with which we can work the same way and thus to simulate the propagation of the beam in the OE.

Detection of the beam escape outside the OE is straightforward. It is sufficient just to check the $z$ coordinate of the beam intersection and test the angle of impact. If the angle of incidence smaller than the angle of aperture the beam goes out of the $\mathrm{OE}$ otherwise it is reflected back and continues to propagate within the OE.
Repetition of a random or systematic generation of the input beam position and direction and the subsequent propagation of the beam by simulation and testing whether the beam reaches properly the tail end of the $\mathrm{OE}$ we get a value of the objective function for a particular geometric shape of the OE. By changing the parameters of the $\mathrm{OE}$ and repeating the simulation we get another value of the objective function. From all tested sets of parameters we finally get the best one for which the value of the objective function is the smallest. Such a set of parameters can then be considered as optimal. It remains to answer the question how to generate values of parameters. A simple but computationally demanding solution is to search for optima using enumerative techniques, when parameters are sampled properly, i.e., the limits of the values of each parameter and dividing is set correctly. The objective function is evaluated for each set of parameters and consecutive simulations. The described numerical model was used for calculation of transmittance $\mathrm{T}$ of four OEs with help of analytical function of their shapes listed in Table 1. Results of model calculation for the most suitable design from tested OEs are shown in Figure 2.

\section{Number of cells in active layer}

As shown in Figure 3, number of cells in active layer depends on size of an area for immobilazation. In the model was hypothesized an assumption that the cells are organized in one layer, cell has a spherelike shape with diameter $1 \mu \mathrm{m}$ and cell is point source. In view of the fact that intensity of emitted light decreases with quadrate of distance

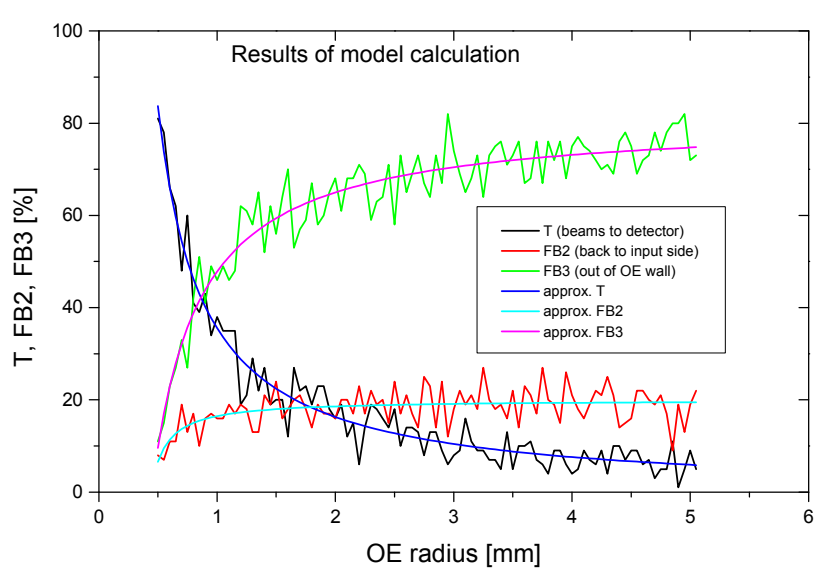

Figure 2: Results of model calculation for OE 3

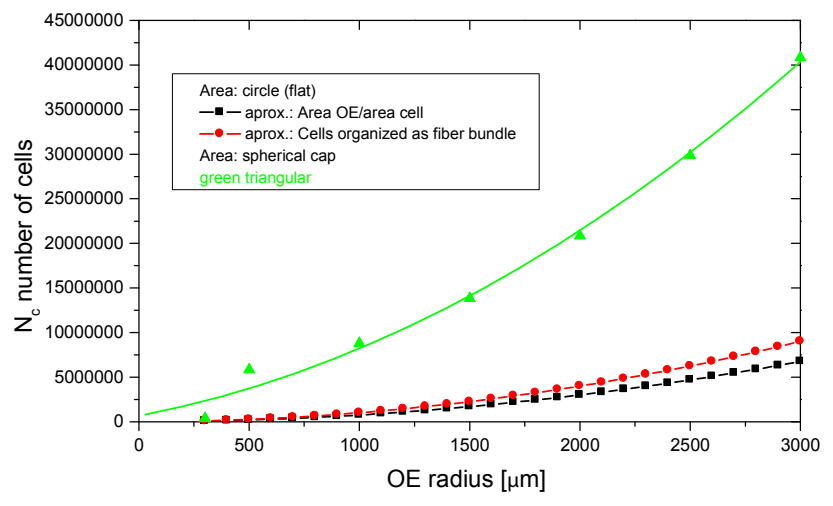

Figure 3: Number of cells as a function of the optical element (OE) radius. 
[7], advantageous place for immobilazatin cells is straight on the surface of $\mathrm{OE}$.

Repetitive inductions of bioluminescence of P. putida TVA8 immobilised by adsorption on modified surface of optical fiber was studied by authors [15].

In this study was applied method of encapsulation of P. putida TVA8 in silica hydrogel described by authors [14]. The active layer has shape of spherical cap (Figure 1) and for this reason we calculated also number of cells on spherical cap area (green line, Figure 3). Authors [16] showed that bioreporters stay alive and active on the surface area of active layer (depth $1 \mathrm{~mm}$ ). We simulated also transmission of rays emmited by biore-porters on the surface of active layer thrue silaca hydrogel to the income of OE. The conditions was chosen for experiments: the active layer (spherical cap) has a refractive index 1.383 (TMOS) and the surrounding environment has a refractive index 1.33 (water). There was calculated that under these conditions $62.54 \%$ of the rays pass to the income part of the $\mathrm{OE}$, the other rays pass through the wall of the active layer into the surrounding environment (Figure 4).

\section{Shape of the optical elements}

In this work the OE were obtained as drops of preforms at the begining of drawing of optical fibers (Figure 5). The shape could be control by temperature in the furnace and the drop mass. Experimental result for shape change coused by different temperature in the furnace

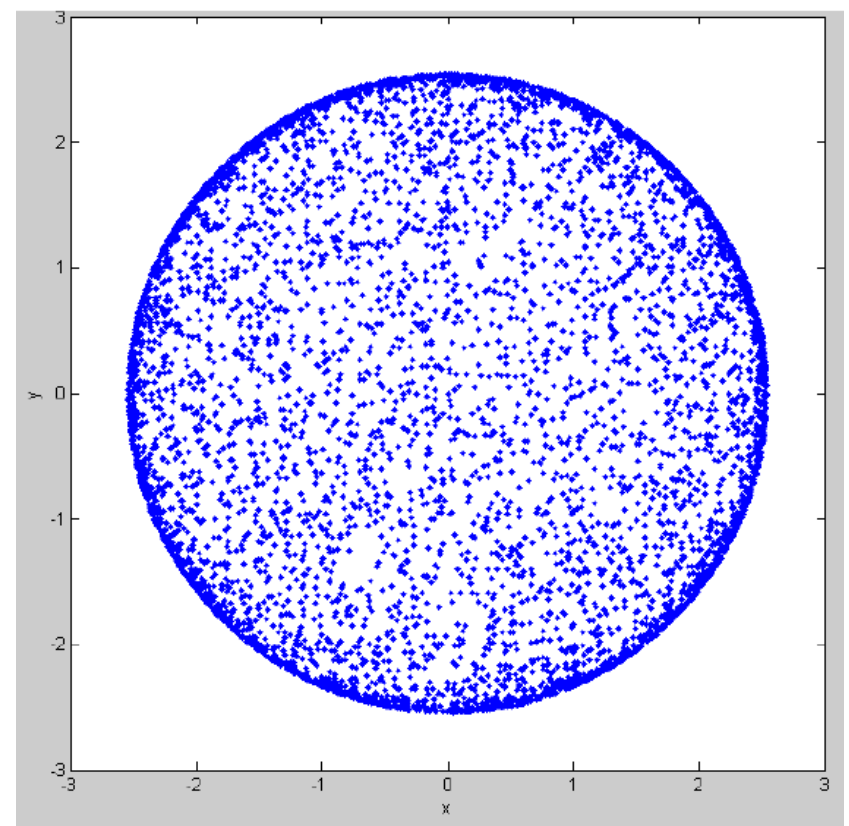

Figure 4: Result of calculation: $62.54 \%$ of the rays emitted by bioreporters on the surfaceof active layer pass to the income part of the optical element.

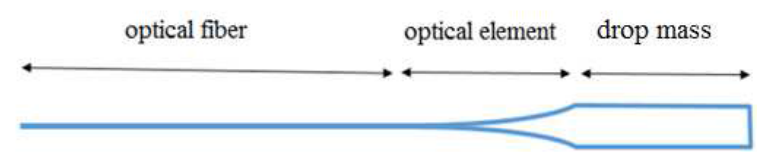

Figure 5: A scheme of optical element manufacture. and length of drop mass, are shown on Figure 6. More precise control of $\mathrm{OE}$ shape enable tapering maschines(Figure 6).

\section{Detection of induced BL}

With aim to demonstrate enhancement of intensity of detected BL by using OE, biore-porter cells $P$. putida TVA8 were immobilized into silica matrix on the ends of PCS fi-ber and OE respectively. Bioluminescence was induced in the YEPS medium with toluene $(26.5 \mathrm{mg} / \mathrm{l})$ [14]. A fourfold increase of detected BL was reached by enlargement of the area with active layer $(\mathrm{OE})$, as is shown on Figure 7. The maximum of 11000 counts/swas reached after 24.5 hours with PCS fiber in contrast to the maximum 45000 counts/s after 13.5 hours for tapered OE. PCS fiber expired within 3 days in contrast to tapered OE what was active for 3 weeks.

\section{Conclusions}

It is seen that OE geometry has significant influence on coupling and transmitting BL to the detector. The goal of this study was development of mathematical model for optimization of OE shape and to compare results of calculations with experimental measurements

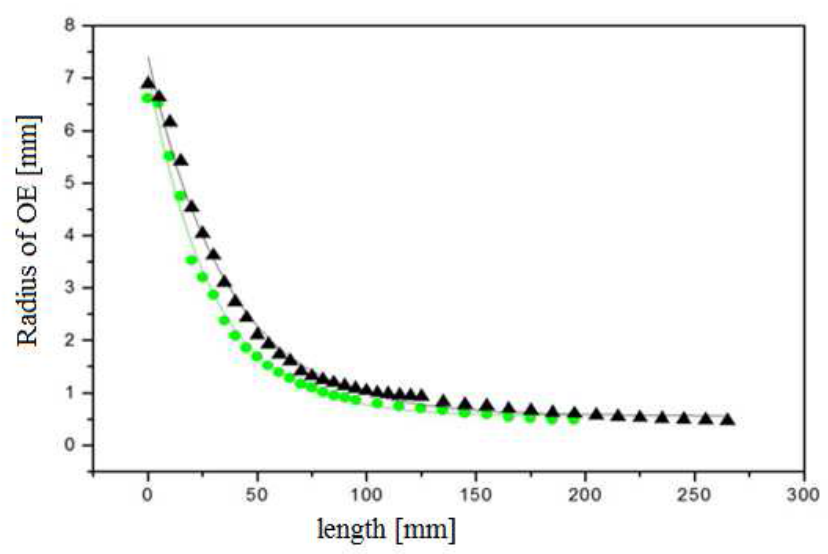

Figure 6: In the graph is shown influence of parametrs on final optical elements shape.The first sample (green line) was obtain under condition (temperature $2050^{\circ} \mathrm{C}$ and $10 \mathrm{~mm}$ of drop mass) different to the second sample (black line, temperature $1970^{\circ} \mathrm{C}$ and $40 \mathrm{~mm}$ of drop mass).

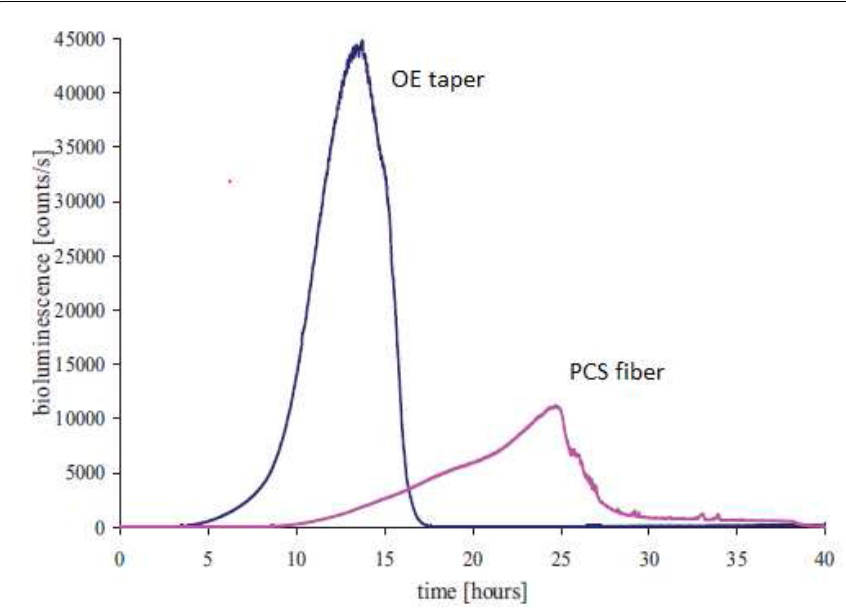

Figure 7: Comparison of detected $\mathrm{BL}$ for measurement set-up with $\mathrm{OE}$ and PCS fiber, respectively. 
of OE that enhance coupled and guided light of the whole-cell optical fiber biosensor. The results of experimental measurement correspond with the results of theoretical calculations. The sixfold increase of the detected BL by tapered OE 3 showed that the geometry of OE 3 is applicable for biosensor design [17]. Biosensing capability was demonstrated with the bioreporters Psuedomonas putida TVA8 in silica gel induced with salicylate. In contrast to another ways of enhancement of detection of bioluminescence as metal-enhanced bioluminescence [18], microluminometrs or bioluminescent integrated circuits [19-21] tapered $\mathrm{OE}$ is advantageous in easiness of preparation and durability that is desirable for in-situ long term monitoring in remote localities. The practical applicability of biosensing with $\mathrm{OE}$ has been already demonstrated by detection of BTEX (benzene, toluene, xylene and ethylbenzene) with reporter $P$. putida TVA8 in water from an influent and an effluent of the wastewater treatment plant and contaminated groundwater [22]. The first results gained with our laboratory biosensor system are shown in Figure 7. Next work will be devoted to study of life conditions the cells in active layer and reproducibility of detected bioluminescence in case of real wastewater samples.

\section{Acknowledgement}

This work was supported by projects SGS14/093/OHK4/1T/17 and CZ.1.07/2.3.00/20.0092 BIO-OPT-XUV (BOX)

\section{References}

1. Bosch ME, Sanchez AJR, Dohas F, Bosch C (2007) Recent Development in Optical Fiber Biosensors. Sensores 7:797-856.

2. Pospisilova M, Kuncova G, Trogl J (2015) Fiber-Optic Chemical Sensors and Fiber-Optic Bio-Sensors. Sensores 15: 25208-25259.

3. Roda A, Guardigli M, Michelini E, Mirasoli M (2009) Bioluminiscence in analytical chemistry and in vivo imaging. Trends in Analytical Chemistry 28: 307-322.

4. Shimomura O (2006) BL: Chemical Principles and Methods. World Scientific Publishing Co. Pte. Ltd, Singapure.

5. Close D, Xu T, Smartt A, Rogers A, Crossley R, et al. (2012) The Evolution of the Bacterial Luciferase Gene Cassette (lux) as a Real-Time Bioreporter. Sensors 12: 732-752

6. Kuncova G, Trogl J (2010) Physiology of microorganisms immobilized into inorganic polymers. In: Morrison DA editor. Handbook of Inorganic Chemistry Research. Nova Science Publishers, NY, USA.
7. Wang Y, Tian W, XiangLi B (2005) Theoretical model of the modulation transfer function for fiber optic taper. SPIE Proceeding, Belingham.

8. Eltzov E, Marks RS (2010) Fiber-Optic Based Cell Sensors. Whole cell sensing systems I: Re-portercells and devices. Book Series: Advances in Biochemical Engineering-Biotechnology. Volume: 117, pp: 131-154

9. Marks RS, Cullen DC, Karube I, Lowe CR, Weetall H (2007) Handbook of Biosensors and Biochips. John Wiley and Sons.

10. Vrbova H, Kuncova G, Pospisilova M (2009) Optical fiber elements for increasing coupling. Trends in Biomedical Engineering, Bratislava, Proceedings of the 8th Czech-Slovak Conference.

11. Fujikura Europe (2017) FSM-45F-LDF.

12. Kuncova G, Pazlarova J, Hlavata A, Ripp S, Sayler GS (2011) Bioluminiscen bioreporter Pseudomonas putida TVA8 as a detector of water pollution. Operational conditions and selectivity of free cells sensor. Ecol Indic 11: 882-887.

13. Meighen EA (1991) Molecular biology of bacterial bioluminiscence. Microbio Rev 55:123-142.

14. Kuncova G, Ishizaki T, Solovyev A, Trogl J, Ripp S (2016) The Repetitive Detection of Toluene with Bioluminescence Bioreporter Pseudomonas putiva TVA8 Encapsulated in Silica Hydrogel on an Optical Fiber. Materials 9: 467.

15. Zajíc J, Bittner M, Brányik T, Solovyev A, Šabata S, et al. (2016) Repeti-tive inductions of bioluminescence of Pseudomonas putida TVA8 immobilised by adsorption on optical fibre. Chemical Papers 70: 877-887.

16. Affi M, Solliec C, Legentilhomme P, Comiti J (2016) Numerical modeling of the dynamic response of a bioluminescent bacterial biosensor. Anal Bioanal Chem 408: $8761-8770$

17. Vrbova H (2010) Influence of geometry and aktive layer of optical elements on sensitivity of biosensor. Czech Technical University in Prague, Faculty of Biomedical Engineering, Czech Republic.

18. Eltzov E, Prilutsky D, Kushmaro A, Marks RS, Geddes CD (2009) Metalenhanced bioluminescence: An approach for monitoring biological luminescent processes. Applied Physics 94: 083901.

19. Marks RS, Cullen DC, Karube I, Lowe CR, Weetall HH (2007) Handbook of Biosensors and Biochips. John Wiley \& Sons, Ltd.

20. Simpson ML, Sayler GS, Patterson G, Nivens D, Bolton EK, et al. (2001) Sensors and Actuators. 72: 135.

21. Close DM, Ripp S, Sayler GS (2009) Reporter Proteins in Whole-Cell Optica Bioreporter Detection Systems, Biosensor Integrations, and Biosensing Applications. Sensors 9: 9147.

22. Adamova N (2009) Development of biosensor with P. putida TVA8. ICT Prague Czech Republic. 